\title{
Optimising operational costs using Soft Computing techniques
}

\author{
Javier Sedano ${ }^{\mathrm{a}}$, Alba Berzosa ${ }^{\mathrm{a}}$, José R. Villar ${ }^{\mathrm{b}}$, Emilio Corchado ${ }^{\mathrm{c}}$, Enrique de la Cal ${ }^{\mathrm{b}}$ \\ ${ }^{a}$ Grupo de Investigación de Inteligencia Artificial y Electrónica Aplicada, Instituto Tecnológico de Castilla y \\ León, Polígono Industrial de Villalonquéjar C/López Bravo, 70, 09001, Burgos, Spain \\ ${ }^{\mathrm{b}}$ Departmento de Informática, Universidad de Oviedo, Campus de Viesques s/n, 33204, Gijón, Spain \\ ${ }^{\mathrm{c}}$ Departamento de Informática y Automática , Universidad de Salamanca, Plaza de la Merced s/n, 37008, \\ Salamanca, Spain
}

\begin{abstract}
A Manufacturing Execution System (MES) consists of high-cost, large-scale, multi-task software systems. Companies and factories apply these complex applications for the purposes of production management to monitor and track all aspects of factory-based manufacturing processes. Nevertheless, companies seek to control the production process with even greater rigour. Improvements associated with an MES involve the identification of new knowledge within the data set and its integration in the system, which implies a step forward to Business Process Management (BPM) systems, from which the users of an MES may gain relevant information, not only on execution procedures but to decide on the best scheduled arrangement. This work studies the data gathered from a real MES that is used in a plastic products factory. Several Artificial Intelligence and Soft Computing modelling methods based on fuzzy rules assist in the calculation of manufacturing costs and decisions over shift work rotas: two decisions that are of relevance for the improvement of the execution system. The results of the study, which identify the most suitable models to facilitate execution-related decision-making, are presented and discussed
\end{abstract}

Keywords: Applied Soft Computing, Artificial Intelligence, Enterprise Resource Planning, Manufacturing Execution Systems

\section{Introduction}

Enterprise Resource Planning (ERP) and Manufacturing Resource Planning (MRP) [74, 113] are wellknown software applications currently used in production and cost optimization in factory plants. These platforms are based on the available Information Technology (IT) frameworks [61, 62]. The increase of the IT presence in industry has resulted in the growth of the aforementioned applications and their integration in production systems $[82,89,94,108$, 119].

Manufacturing Execution Systems (MES) are IT systems that are used for management resource planning: equipment, employees and inventories [18, 112].

An MES may be implemented in the context of a production control system or a manufacturing monitoring and supervisory system. In the former case, the objective is to provide the company with a research laboratory for products and processes, whereas in the latter case, the MES is a computer-aided system that assists with decision-making processes that relate to manufacturing.

However, designing and deploying a user-friendly MES which fulfils the above-mentioned objectives represents a significant challenge, owing to a large extent to the complexity of modern production systems, plants and products $[28,90]$.

Soft computing $[6,19,21,32,34,61,80,111]$ is a collection or set of computational techniques in machine learning $[1,4,42,86]$, such as artificial neural networks $[3,7,8,11,12,13,15,40,43,47,48,49$, $31,55,56,57,64,77,78,79,83,101,104,109]$, genetic algorithms $[9,10,16,22,23,26,29,60,67$, $70,72,73,95,96,97,98]$, fuzzy systems [5, 6, 20, $54,58,68,71,87,100,121,114]$, simulated annealing algorithms [122], spiking neural networks [2, 41,

*Corresponding author. E-mail: villarjose@uniovi.es. 
$59,76,91,99,106,107]$, case-based reasoning [65, $105,116]$, and swarm intelligence $[35,36,37,88$, $115,118]$, which investigate, simulate and analyze very complex issues and phenomena concerned with problems in which tractability, robustness and uncertainty are significant factors [46]. There is also a significant number of recent articles on developing hybrid systems through the integration of various soft computing techniques $[44,50,63,81,110,117]$ or integration with other techniques such as wavelets $[14,39,92]$.

This study applies several soft computing techniques and analyses their results. Its aim is to obtain classification models that, according to different manufacturing conditions -machines, products, stoppage time, run time, micro stoppage time...-, support staff at a plastic products factory with budgeting costs management- and operator shift rotas. Nevertheless, the final and mid-term aim of this study is to extend its scope and integrate the models into the company's MES, in order to enhance the capability to estimate real operational costs under the best conditions for execution. Section 2 describes the problem scenario, and subsequent subsections consider the objective of the study and similar approaches in the literature. Section 3 explains the fundamentals of the different methods in use, while Section 4 deals with the methodology and techniques to obtain the models and also includes a description of the experimentation and a discussion of the results. Finally, the conclusions are presented and future lines of work outlined.

\section{A real case study: a plastic products factory}

This investigation examines the MES of a plastic products factory in Spain that manufactures different products: tubes, (polypropylene) sheets and (garbage) bags, among others. Its production process is divided into a storage area, an extrusion area and a printing and clothing area.

Figure 1 depicts the schema of the plastic bags factory where the production system is totally supervised and monitored.

Each machine has its own control system based on Programmable Logic Controllers (PLC). There are up to 75 machines, each producing a range of different products. There are also several Human Machine Interfaces (HMIs) connected to an Ethernet network and a Data Acquisition System (DAQ) that collects various process signals, pressures and temperatures, among others.

The operators control the machines that are programmed to manufacture the product. Finally, the monitoring and supervisory computers that are connected to the network request information from the PLCs and DAQs. The entire network is known as the Manufacturing Control System (MCS).

A real data set was compiled with the aim of discovering new knowledge from data that could be integrated in the MES system. The main idea is to improve the capacity of the staff to estimate operational costs and production scheduling in the factory. Only a small amount of data was available, as the company had only recently begun to store data in a database management system.

Firstly, the production dynamics characteristics should be determined to integrate the MES into this scenario. To do so, the available data set has to be pre-processed and its relevant variables and partitions should be extracted according to manufacturing conditions. Once the manufacturing dynamics data have been pre-processed, a production operations model may be formulated [25].

\subsection{Analysis of the objectives}

As stated before, the final objective of this study is to obtain hidden know-how in the data set and to show how it can be incorporated as one or more models in the real MES to support factory staff in tasks relating to budgeting - costs management- and operator scheduling. Consequently, all available data sets in the MES from the MCS should be examined in the design of the final database.

The working method had been analysed in order to propose a solution for the first objective. Although there is a product catalogue, the client can also order customized products. When a client orders a product, its characteristics are all defined, i.e.: gross material usage, product specifications etc. Then a staff member analyses the requirements, assigns the job to a certain machine chain and estimates its cost. This process is as yet not automated, so the employee needs to analyze several plots and reports before assigning a machine chain. The challenge throughout these steps is to develop a classifier for the cost level that is associated with the configuration of a product that will be manufactured, the client's specifications and the proposed machine. This will allow bidding in accordance with the real cost of the productive operations. 
A further problem to address is the assignment of operators for machining certain products, so that staff can decide on the best shift schedules.

In this case, the inputs to the model should be the product, client, machine and operator identifiers, among others. The challenge is to classify the effectiveness of the configuration.

The greater the interpretability of the models, the better prepared it is to perform to a high degree of accuracy. This may be demonstrated through a comparison with non-interpretable models.

One of the difficulties in developing a broad solution to this problem is that the literature only contains different ad hoc solutions to specific problems [18, $25,27,28,90]$. A solution may be proposed based on these ideas, but it is not a clear extension of previously published works which would satisfy the objectives of the study.

The complex task of integrating the different systems should consider open architectures and clearly defined procedures for interchanging information. These procedures are currently available as open standards; different structures for the design and integration of the MES are discussed in [27].

The problem of integrating the MES, the data warehouse, online analytical processing and data mining systems have previously been discussed in [25], where decision trees were used to extract, learn and model the required knowledge. In [18], integrations are proposed in which the customer's system must not have access to the MES data directly. Consequently, once suitable models were chosen, their integration within the MES should consider the above-mentioned ideas. The analysis of the data and the study of suitable models is the main purpose of this research, while the selection of suitable models and their integration within the MES is left for a future occasion.

\section{Prototyping and Experimentation}

Having collected the available data set, several tasks should be performed. Firstly, the data set has to be analyzed and pre-processed in order to determine whether there are any dependent variables. An analysis is also necessary to decide whether the data should be normalized, and whether a partition of the class variable is needed.

As stated in previous sections, two problems had to be solved and a model for each task should be ob- tained: a model for assisting with budgeting and a model for shifting the operators to each machine.

This Section refers to all the tasks that are needed to obtain the models for both problems. Firstly, data harvesting is outlined. Subsection 3.2 refers to data mining and the knowledge extraction tools and techniques that are proposed, while Subsection 3.3 looks at data set pre-processing. Subsections 3.4 and 3.5 describe the modelling of the budgeting problem and the shift work rota and scheduling problem, respectively. Finally, the results are discussed in Subsection 3.6.

\subsection{Data acquisition from the MCS}

The MCS framework is the source of the data to develop the modelling tasks. In this study, data availability is restricted to data that is currently available to staff with a decision-making role. This restriction is because the effectiveness of these models should be evaluated with the relevant staff, who might otherwise draw their own conclusions from the results.

Consequently, the available data is fixed to a predetermined feature set, which includes the following variables:

- Client: the name of the company that requests the manufacture of certain products, which generates a manufacturing order number for the product.

- Product: identification of the product that will be manufactured.

- Machine: the identifier of the machine that was assigned to a manufacturing order.

- Day: the date when manufacturing began.

- Operator: the operator or operator team that manufactures a product per day and per machine. This is a non-atomic field.

- Units: the amount of product units manufactured by a machine for a manufacturing order.

- Kg. of units produced: total units manufactured in $\mathrm{kg}$.

- Discarded units: the number of discarded product units in a machine. Units discarded represent quality errors.

- Kg. of discarded units: manufactured units discarded in $\mathrm{kg}$.

- OEE: overall equipment effectiveness index.

- Time spent in production: the sum of the operating time (Tm) -the run time-, the stoppage time (Ts) and micro-stoppage time (T $\mu \mathrm{s})$ - of the machine-.

- Run time (Tm): the total time spent manufacturing a product. 
- Stoppage time (Ts): total machine stoppage time during product manufacturing.

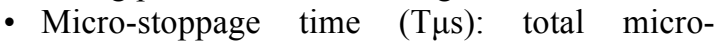
stoppages time during the manufacture of the product.

- Stoppages: the number of stoppages that take place during the manufacture of the product.

- Micro-stoppage: the number of micro-stops during the manufacture of the product.

\subsection{Data mining and Knowledge extraction issues}

Knowledge Extraction based on Evolutionary Learning (KEEL) software [17] was used in all the experimental and modelling stages. KEEL software is a research and educational tool for modelling data mining problems which implements more than one hundred algorithms, including classification, regression, clustering, etc.

Moreover, it includes data pre-processing and post-processing algorithms, statistical tests and reporting facilities. Finally, it has a module for data set analysis and formatting, which was used for the first task in this experiment.

There are several data mining and knowledge extraction tools, such as the Weka [45] and the Orange [51] suites, all of which could be valid for this experimentation. An exhaustive list of these kinds of suites can be found in [66].

Several different techniques were selected to extract knowledge from the data set gathered from the MES. Fuzzy Rule-Based Systems and Decision Trees are considered suitable for IT support tools because of the interpretability of their models $[69,120]$. Several techniques are also able to manage the type of data that is available.

Different techniques were used to compare the results and the viability of the models. The statistical methods included Quadratic Discriminant Analysis (QDA) [75], the Multinomial Logistic regression model with a ridge estimator (LOG) [24], the Kernel Classifier [75], and the K-nearest neighbour [33].

The fuzzy rule-based methods included the Fuzzy Adaboost rule learning method (ADA) [53], the Fuzzy GA-P algorithm (FGAP) [93] and the Ishibuchi Hybrid Fuzzy GBML (HFG) [52]. Finally, two well-known decision tree and decision tree rulebased methods were used: the C4.5 [84] and C4.5 rule-based methods. (C45R) [85].

In the QDA algorithm, the cost of classifying an example $X$ with class $k$ is calculated through Eq. (1), where $\pi$ is the unconditional prior class $k$ probability estimated from the weighted sample, and $\mu$ and $\Sigma_{k}$ are, respectively, the mean population vector and the covariance matrix for the $k$ class. Hence, an example $X$ is assigned with the minimum cost class as stated in Eq. (2).

$$
\begin{gathered}
d_{k}(X)=\left(X-\mu_{k}\right)^{T} \sum_{k}^{-1}\left(X-\mu_{k}\right) \\
+\ln \left|\Sigma_{k}\right|-2 \cdot \ln \pi_{k} \\
d_{\widehat{k}}=\min _{1 \leq k \leq K}\left\{d_{k}(X)\right\}
\end{gathered}
$$

The LOG algorithm is based on the standard logistic regression. The probability that class $\mathrm{k}$ correctly classifies the example $X=\left\{X_{1}, \ldots, X_{p}\right\}$ is calculated following Eq. (3), where the parameter $\beta=\left\{\beta_{1}, \ldots, \beta_{\mathrm{p}}\right\}$ is estimated, i.e., with the maximum likelihood estimation obtained by maximizing Eq. (4). The class with higher probability is chosen to label the example.

$$
\begin{array}{r}
p(k \mid X)=\frac{\exp \left(\sum_{j=1}^{p} \beta_{j} X_{j}\right)}{1+\exp \left(\sum_{j=1}^{K} \beta_{j} X_{j}\right)} \\
l(\beta)=\quad \sum_{k}(k \cdot \log p(k \mid X) \\
+\neg k \cdot \log (1-p(k \mid X)))
\end{array}
$$

The Kernel method is a Bayes rule classifier that, as stated in [38], uses a "non-parametric estimation of the density functions through a Gaussian kernel function." In the KEEL software, an ad-hoc method performs covariance matrix tuning. In contrast, the $\mathrm{K}$ nearest neighbour method classifies the example $X$ with the majority class in $\mathrm{K}$ examples of the data set at the shortest distance from $X$. Note that the use of the KNN implies that a metric is defined in the space to measure the distance between examples.

The Fuzzy Adaboost method is based on boosting $\mathrm{N}$ weak fuzzy classifiers (that is, $\mathrm{N}$ unreliable fuzzy classifiers are weighted according to their reliability) so that the whole outperforms each of the individual classifiers. Moreover, each example in the training data set is also weighted and tuned in relation to the evolution of the whole classifier.

The GAP is a Fuzzy Rule-Based Classifier trained using the Genetic Programming principles but using the Simulated Annealing algorithm to mutate and to evolve both the structure of the classifier and the parameters. The whole Fuzzy Rule set will evolve in each iteration. 
The Ishibuchi Hybrid Fuzzy Genetic Based Machine Learning method represents a Pittsburgh style genetic learning process which is hybridized with the Michigan style evolution schema: after generating the $\left(N_{\text {pop }}-1\right)$ new Fuzzy Rule sets, a Michigan style evolutionary scheme is applied to each of the rules for all the individuals. Recall that each individual is a complete Fuzzy Rule set.

Finally, the C4.5 algorithm is a well-known decision-tree method based on information entropy and information gain. A node in the decision tree is supposed to discriminate between examples of a certain class based on a feature value. At each node, the feature that produces the higher normalized information gain is then chosen. In the case of C4.5R, the decision tree is presented as rules, where each node in the path from the root to a leaf is considered an antecedent of the rule. These rules are then filtered to eliminate redundant or equivalent ones.

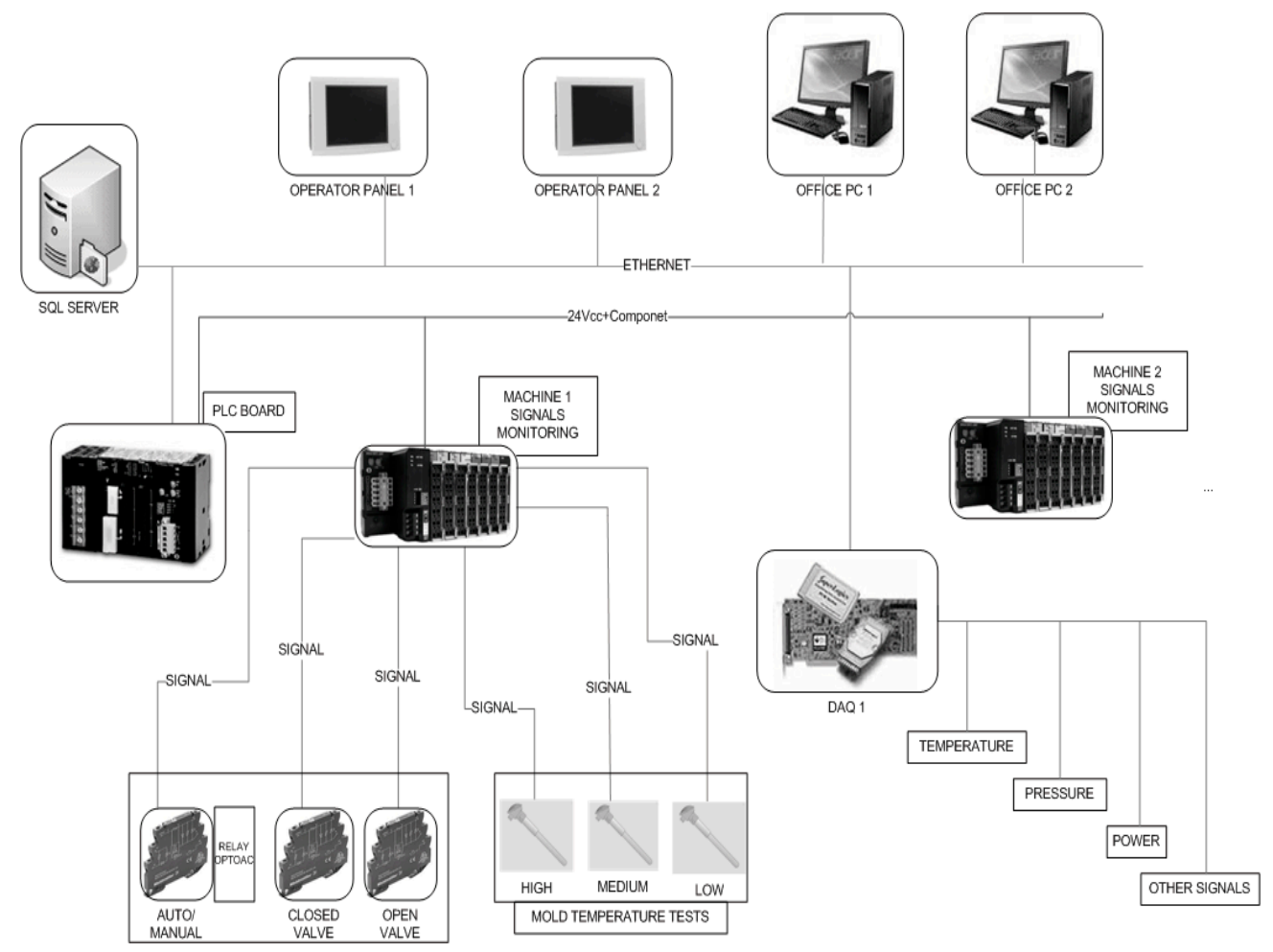

Fig. 1. A schematic diagram of the MES installed in the plastic products factory.

The PLCs which control each machine and the DAQs and HMIs connected through the field network constitute the MCS.

\subsection{Pre-processing and partitioning of the data set}

The budgeting problem and the shift work rota and scheduling problem require different data-set preprocessing and partitioning steps.

For the budgeting problem, a data set was gathered from the IT framework that amounted to $1,471 \mathrm{ex}-$ amples, containing the available historical records that comprised 22 input variables and included the features mentioned in Subsection 3.1.

After analyzing the original data set it was found that most of the examples corresponded to the tuning of the plant, and could therefore be discarded. In ad- dition, a large quantity of totally erroneous samples were also found, which also had to be discarded.

The output variable is a class variable that represents the level of the production cost. The staff at the company chose the two different partitioning schemes. The first partition is a three-class problem, with the labels \{Low, Medium, High .

In the second partition scheme, the problem is divided in a two-class problem, with the labels Low, $\neg$ Low $\}$. The examples classified as $\neg$ Low have also been classified as $\{$ Medium, High $\}$. In this second partitioning scheme it is assumed that two classifiers should be obtained: the first one discriminates the 
Low class and a second (only obtainable with the $\neg$ Low examples) discriminates the Medium class.

Several relationships were found between the features of the data set; i.e.: the one between the number of faulty units and the weight of discarded material.

Consequently, the original data set was reduced from 1,471 examples to the final data set containing 168 examples. This final data set has 4 input variables: product identification, machine identification, client identification and the number of units to produce.

In the case of the shifting and scheduling problem the original data set gathered from the IT framework contained 2,792 examples, including historical records of up to 30 input variables. The same two partitioning schemes used for the budgeting problem were applied to the scheduling problem. Once again, the staff proposed the labels for each example. The labels for the scheduling problem are $\{$ Good, Compromise, Invalid $\}$ and $\{$ Good, $\neg$ Good $\} /\{$ Compromise, Invalid $\}$.
The final data set, after pre-processing and filtering and the relationships discovery process, contained 1215 samples with 2 input variables.

\subsection{Modelling the budgeting problem}

The objective of this model is to determine whether an association between a product and a specific machine will generate a certain cost level.

The methods described in the subsection 3.1 were used to obtain the classifiers. The two partitioning schemes were analyzed and modelled for each case. As there were so few examples, a 10-fold crossvalidation scheme was selected and performed in a KEEL environment. The classifying error was used as the index for choosing the best algorithm.

The parameters of each method are shown in Table 1 , alongside their corresponding acronyms.

Table 1

Parameters used in the experimentation for modelling

\begin{tabular}{|c|c|}
\hline Method & Computer-based model for budgeting \\
\hline Kernel Classifier (KC) & Sigma Kernel $=0.01(\mathrm{KC} 01)$ or $0.05(\mathrm{KC} 05)$ \\
\hline K nearest Neighbour (KNN) & $\begin{array}{l}\text { Distance function: Euclidean; K value }=1 \\
\text { (KNN1) or } 3 \text { (KNN3) }\end{array}$ \\
\hline $\begin{array}{l}\text { Multinomial Logistic regression } \\
\text { model with a ridge estimator (LOG) }\end{array}$ & Ridge value $=10^{-8} ;$ iterations $=1$ \\
\hline Fuzzy AdaBoost (ADA) & Number of labels $=3 ;$ number of rules $=8$ \\
\hline $\mathrm{C} 4.5$ & Pruned; confidence $=0.25 ; 2$ instances per leaf \\
\hline Rule based C4.5 & $\begin{array}{l}\text { Threshold }=10 \text {; confidence }=0.25,2 \text { instances per } \\
\text { leaf }\end{array}$ \\
\hline $\begin{array}{l}\text { Ishibushi Hybrid Fuzzy GBML } \\
\text { (HFG) }\end{array}$ & $\begin{array}{l}\text { number of fuzzy rules }=35 ; \text { number of fuzzy rule } \\
\text { sets }=200 ; \text { crossover probability }=0.9 ; 1,000 \\
\text { generations; probability of Michigan iteration }= \\
0.5\end{array}$ \\
\hline Fuzzy GAP (GAP) & $\begin{array}{l}\text { number of labels }=7 ; \text { number of rules }=35 ; \text { popu- } \\
\text { lation size }=50 ; 2 \text { islands; iterations }=1,000 ; \\
\text { tournament size }=4 ; \text { mutation and migration prob- } \\
\text { abilities }=0.01 \text { and } 0.001 ; \text { mutation amplitude }= \\
0.1 ; 8 \text { niches; intra-niche migration, gp and ga } \\
\text { crossover and mutation probabilities }=0.75,0.5 \\
\text { and } 0.5 ; \text { length of the gap chain }=10 ; \text { tree height }= \\
8\end{array}$ \\
\hline
\end{tabular}




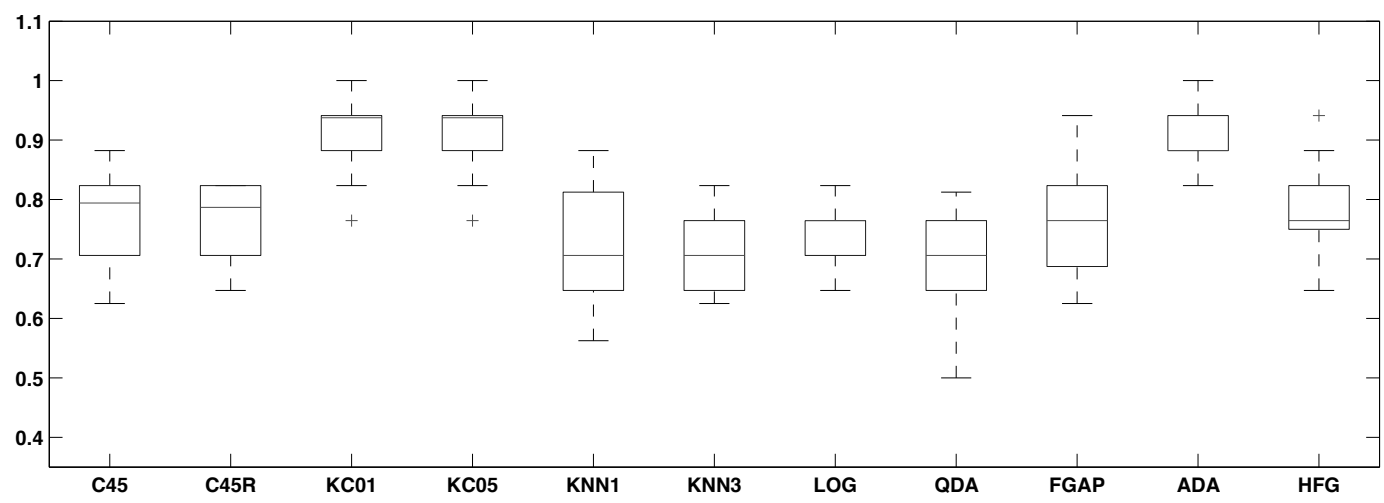

Fig. 2. The computer-based model for budgeting: Experimental results for the two classes of problems, classes $\{$ Low, $\neg$ Low $\}$. As may be seen, the best results are obtained for the Kernel and the Fuzzy AdaBoost methods.

Table 2

The budgeting computer-based model: mean results obtained for the classifiers in the $\{$ Low, $\neg$ Low $\}$ and the $\{$ Medium, High $\}$ two-class experiments.

GCE: Global Classification Error; SGCE: standard deviation of the GCE; CC: percentage of correctly classified examples.

\begin{tabular}{|lllllll|}
\hline & \multicolumn{3}{c}{ Low, $\neg$ Low } & \multicolumn{3}{c|}{ Medium, High } \\
\hline Method & GCE & SGCE & CC & GCE & SGCE & CC \\
\hline C4.5 & 0.2276 & 0.0748 & 0.7724 & 0.1018 & 0.1220 & 0.8982 \\
\hline C4.5R & 0.2324 & 0.0620 & 0.7676 & 0.1018 & 0.1230 & 0.8982 \\
\hline KC01 & 0.0949 & 0.0651 & $\mathbf{0 . 9 0 5 1}$ & 0.0949 & 0.0651 & 0.9051 \\
\hline KC05 & 0.1143 & 0.0879 & 0.8857 & 0.1018 & 0.0758 & 0.8982 \\
\hline KNN1 & 0.2860 & 0.1002 & 0.7140 & 0.2464 & 0.1746 & 0.7536 \\
\hline KNN3 & 0.2857 & 0.0695 & 0.7143 & 0.3107 & 0.2295 & 0.6893 \\
\hline LOG & 0.2504 & 0.0530 & 0.7496 & 0.0750 & 0.0829 & 0.9250 \\
\hline QDA & 0.3040 & 0.0858 & 0.6960 & 0.0911 & 0.0820 & 0.9089 \\
\hline FGAP & 0.2335 & 0.0973 & 0.7665 & 0.0893 & 0.0810 & 0.9107 \\
\hline ADA & 0.0945 & 0.0598 & $\mathbf{0 . 9 0 5 5}$ & 0.0500 & 0.0829 & $\mathbf{0 . 9 5 0 0}$ \\
\hline HFG & 0.2206 & 0.0800 & 0.7794 & 0.0750 & 0.0829 & 0.9250 \\
\hline
\end{tabular}

The results from the two-class partitioning scheme experiments are presented in Table 2, Figure 2 and Figure 3. As may be seen, the best models were the Kernel method and Fuzzy AdaBoost, which are capable of correctly classifying more than $90 \%$ of the actual samples.

The three-class partitioning scheme experiments present a poorer performance for all the algorithms, except for the kernel methods, which keep track of the problem (see Table 3). They are capable of correctly classifying close to $90 \%$ of the actual samples. The drop in algorithm performance may be due to the small size of the data set. Moreover, it appears that greater effort may be needed at the pre-processing stage, i.e.: discretization of all variables, analysis of different missing values and techniques, etc. 


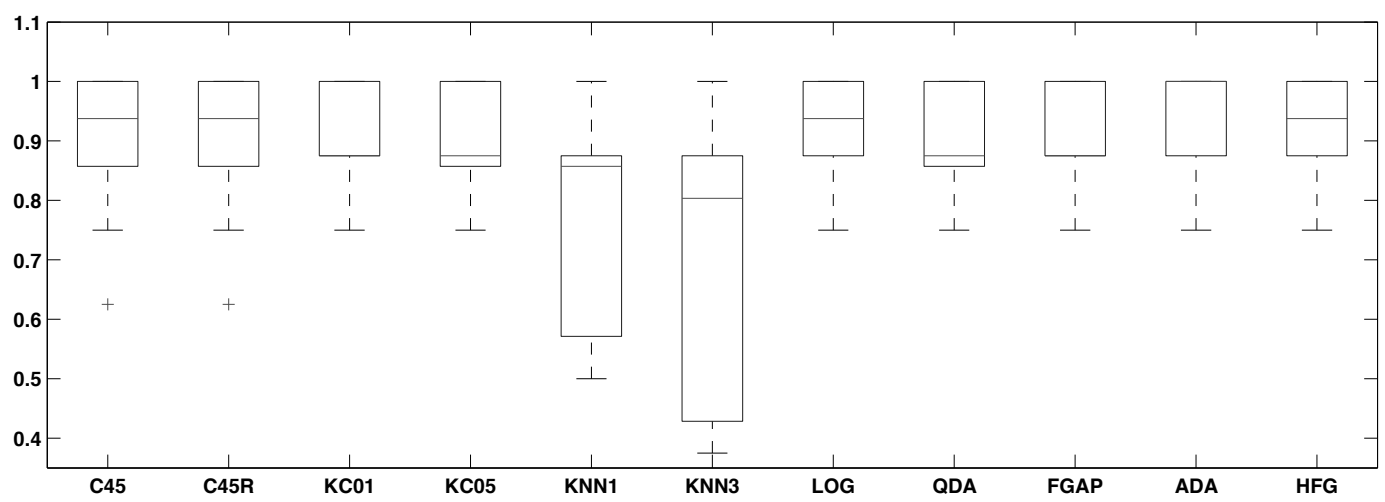

Fig. 3. The budgeting computer-based model: Experimental results for the \{Medium, High $\}$ two-class problem. The best results were obtained by the Kernel and the Fuzzy AdaBoost methods.

Table 3

The computer-based model for budgeting: mean results obtained for the classifiers in the \{Low, Medium, High \} three-class experiments. GCE: Global Classification Error; SGCE: standard deviation of the GCE; CC: percentage of correctly classified examples.

\begin{tabular}{|l|l|l|l|}
\hline & \multicolumn{3}{|c|}{ Low, Medium, High } \\
\hline Method & GCE & SGCE & CC \\
\hline C4.5 & 0.2974 & 0.0441 & 0.7026 \\
\hline C4.5R & 0.3103 & 0.0967 & 0.6897 \\
\hline KC01 & 0.1077 & 0.0648 & $\mathbf{0 . 8 9 2 2}$ \\
\hline KC05 & 0.1077 & 0.0531 & $\mathbf{0 . 8 9 2 3}$ \\
\hline KNN1 & 0.3445 & 0.0796 & 0.6555 \\
\hline KNN3 & 0.3684 & 0.1120 & 0.6316 \\
\hline LOG & 0.2434 & 0.0840 & 0.7566 \\
\hline QDA & 0.3338 & 0.0857 & 0.6662 \\
\hline FGAP & 0.4118 & 0.0975 & 0.5975 \\
\hline ADA & 0.1783 & 0.0785 & 0.8217 \\
\hline HFG & 0.3857 & 0.0799 & 0.6143 \\
\hline
\end{tabular}

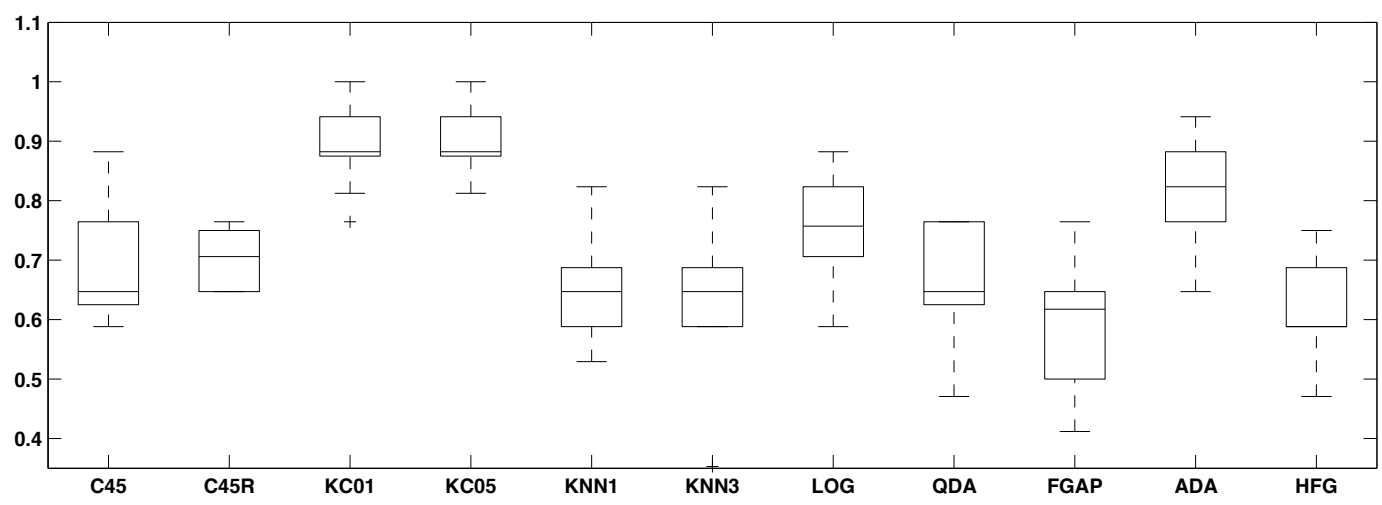

Fig. 4. The budgeting computer-based model: Experiment results for the \{Low, Medium, High\} three-class problem. The best results were obtained by the Kernel methods, then by the Fuzzy AdaBoost method.

\subsection{Modelling the scheduling problem}

The aim of this model is to determine whether an association between a product and a specific machine and operator would be suitable.
The methods described in Subsection 3.2 have been used again to obtain the classifiers; Table 1 shows the parameters used for each of the methods, along with their corresponding acronyms.

The two partitioning schemes were also analyzed and modelled for each case. The 10-fold cross- 
validation schema was selected and performed in a used as the index to choose the best algorithm. KEEL environment. Again, the classifying error was

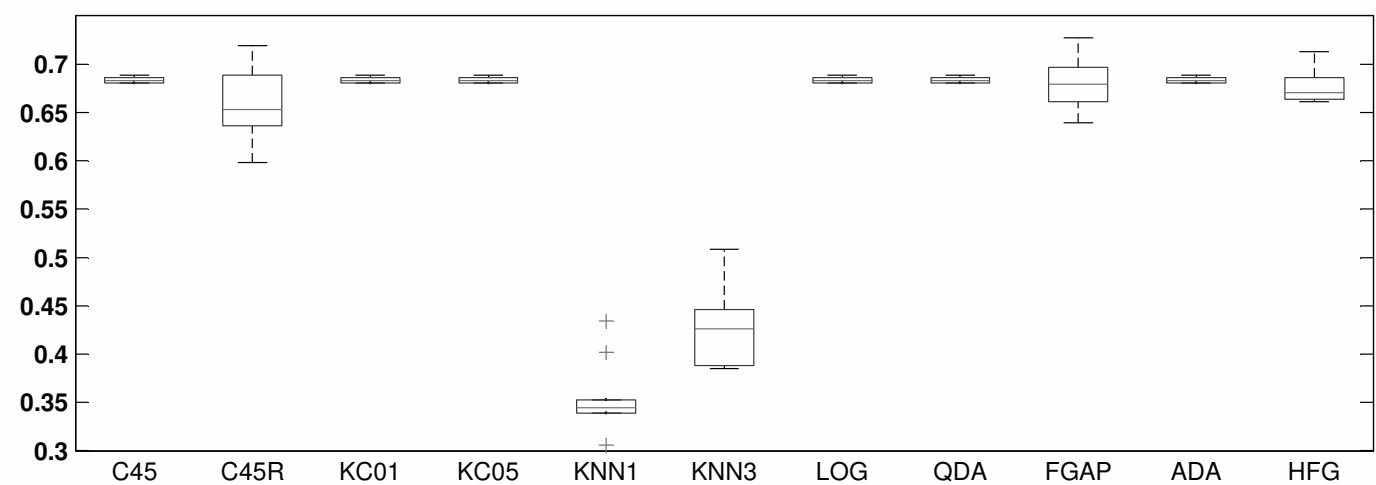

Fig. 5. The scheduling problem model: Experiment results for the $\{\mathrm{Good}, \neg \mathrm{Good}\}$ two-class problem. As can be seen, only the K-nearest neighbour methods fail to behave properly.

Table 4

The scheduling problem model: mean results for the classifiers in the $\{$ Good, $\neg$ Good $\}$ and the $\{$ Compromised, Invalid $\}$ two-class experiments. GCE: Global Classification Error; SGCE: standard deviation of the GCE; CC: percentage of correctly classified examples.

\begin{tabular}{|lllllll|}
\hline & \multicolumn{3}{c}{ Good, $\neg$ Good } & \multicolumn{2}{c|}{ CCompromised, Invalid } \\
\hline Method & GCE & SGCE & CC & GCE & SGCE & CC \\
\hline C4.5 & 0.3166 & 0.0032 & $\mathbf{0 . 6 8 3 4}$ & 0.4453 & 0.0016 & 0.5547 \\
\hline C4.5R & 0.6555 & 0.3445 & 0.0340 & 0.4935 & 0.0553 & 0.5065 \\
\hline KC01 & 0.3166 & 0.0032 & $\mathbf{0 . 6 8 3 4}$ & 0.4453 & 0.0016 & $\mathbf{0 . 5 5 4 7}$ \\
\hline KC05 & 0.3166 & 0.0032 & $\mathbf{0 . 6 8 3 4}$ & 0.4453 & 0.0016 & $\mathbf{0 . 5 5 4 7}$ \\
\hline KNN1 & 0.6456 & 0.0347 & 0.3544 & 0.5427 & 0.0115 & 0.4573 \\
\hline KNN3 & 0.5757 & 0.0365 & 0.4243 & 0.5295 & 0.0383 & 0.4705 \\
\hline LOG & 0.3166 & 0.0032 & 0.6834 & 0.4537 & 0.0182 & 0.5463 \\
\hline QDA & 0.3166 & 0.0032 & 0.6834 & 0.4669 & 0.0313 & 0.5331 \\
\hline FGAP & 0.3224 & 0.0253 & 0.6776 & 0.4489 & 0.0358 & 0.5511 \\
\hline ADA & 0.3166 & 0.0032 & 0.6834 & 0.4573 & 0.0148 & 0.5427 \\
\hline HFG & 0.3232 & 0.0165 & 0.6768 & 0.4670 & 0.0445 & 0.5330 \\
\hline
\end{tabular}

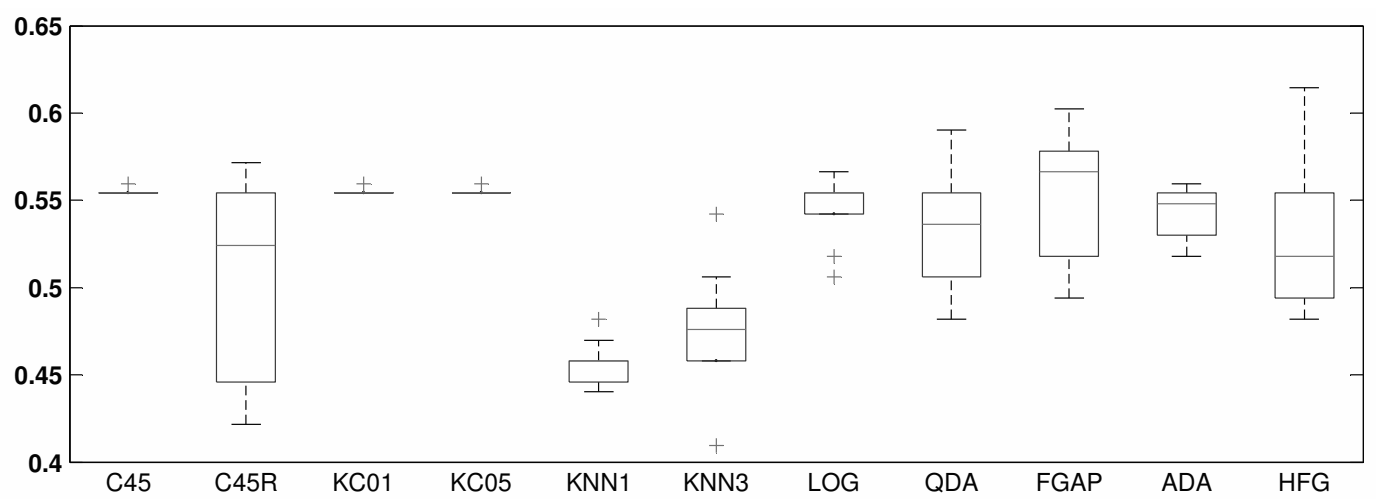

Fig. 6. The scheduling problem model: Experimental results for the \{Compromised, Invalid\} two-class problem.

The worst methods were the K-nearest neighbour and classifier confidence measures were poor.

Table 5

The scheduling problem model: mean results for the classifier in the \{Good, Compromised, Invalid\} three-class experiment. GCE: Global Classification Error; SGCE: standard deviation of the GCE; CC: percentage of correctly classified examples.

\begin{tabular}{|llll|}
\hline & \multicolumn{3}{l|}{ Good, Compromised, Invalid } \\
\hline Method & GCE & SGCE & CC \\
\hline C4.5 & 0,5994 & 0,0376 & 0,4006 \\
\hline
\end{tabular}




\begin{tabular}{|llll|}
\hline C4.5R & 0.6357 & 0.0324 & 0.3643 \\
\hline KC01 & 0.6209 & 0.0025 & 0.3791 \\
\hline KC05 & 0.6160 & 0.0160 & 0.3840 \\
\hline KNN1 & 0.6883 & 0.0147 & 0.3117 \\
\hline KNN3 & 0.6917 & 0.0205 & 0.3083 \\
\hline LOG & 0.6308 & 0.0247 & 0.3692 \\
\hline QDA & 0.6250 & 0.0216 & 0.3750 \\
\hline FGAP & 0.9416 & 0.0241 & 0.0584 \\
\hline ADA & 0.6226 & 0.0278 & 0.3774 \\
\hline HFG & 0.6118 & 0.0324 & 0.3882 \\
\hline
\end{tabular}

The results from the first experiment are presented in Table 4, Figure 5 and Figure 6. As may be seen, it correctly classifies almost the $70 \%$ of the examples, when discriminating between the Good and $\neg$ Good classes. In the second three-class problem, the results obtained are quite discouraging (see Table 5) as explained in the next sub-section and, consequently, no graphics results are shown.

\subsection{Discussion}

The results from the scheduling problem are not as suitable as expected, mainly due to the low quality of the data; in other words, the information contained in the data is not informative enough. One of the reasons for this behaviour emerged when presenting the results to the company: the examples in the data set were not independent samples.

The case of shift work rotas with two operators to manufacture a product on the same machine was treated as two examples with the same data, but they involve different operator identification information. Consequently, if one of the two operators performed better than the other, there was no way to distinguish between them. Unfortunately, this was quite common in the data set, which implied poorer results for the classifiers.

An important conclusion may be drawn from this experiment: the data set should be more informative and representative of the problem, if better models have to be generated. The company should rely on an in-depth analysis of the available data and measurements, but it is also necessary to study the relationships between the variables under consideration, i.e. using Cooperative Maximum Likelihood Hebbian Learning (CMLHL) [30] as shown in [102, 103].

For the case of the budgeting problem, the above mentioned Fuzzy Ada-Boost and the Kernel methods are the more suitable algorithms. Improvements to the FGAP and the HFG algorithms are also suggested, in order to reduce the standard deviation.

It could be said that these two methods may improve their performance with a better definition of their parameters (population and sub-population sizes, number of islands, etc.) and if they had a larger number of generations.

It is worth remarking that this is especially interesting in the case of a two-class problem, the performance of which is better than it is in the three-class problem.

Obviously, there is not enough information to obtain good models in the budgeting problem for the three-class problem.

As an example, the number of units to be produced is somewhat dependent on the machine, as each machine has a maximum production rate. But this data was not used in the experiment, so it was not possible to normalize those variables, which in turn, reduced the quality of the classifier.

If a model should be chosen for the shifting and scheduling problem, it could be said that the interpretable models represent the best option as they all perform with a similar error distribution but they allow the staff to analyse their proposed decisions. In the case of the shifting and scheduling problem, results in Table 4 show that the C4.5 and the kernel methods are the most suitable for the first problem (the computer-based support model for budgeting), as presented in [25]. Nevertheless, the relatively low accuracy of all the algorithms suggest that we redesign the way the data set is formed and repeat all of the modelling steps. The improved data set, with larger amounts of information on the process, will surely enhance the algorithms results.

\section{Conclusions}

This interdisciplinary research presents how to analyze and discover knowledge within the data set in an MES for its integration in the system, to improve factory capacity and support the staff of a plastic products factory with budgeting -costs managementand shift work rotas for operators .

A classification model for the budgeting problem was solved through the application of different com- 
puting techniques. The selected models are able to classify the different levels of costs. However, the scheduling problem cannot be solved with the same approach and the same initial data collection.

It is worth remarking on the importance of analyzing the data that has to be gathered before performing the experiments. The example of the scheduling problem clearly illustrates this sort of situation.

An experimental design about a clear list of the objectives to be accomplished by the MES should be prepared prior to the collection and analysis of relevant data.

Future work will include modelling using different heuristics, i.e. multi-objective solutions and simulated annealing, with the aim of solving problems that can be integrated within the enterprise resource planning. More data should be gathered from the plant, such as machine operating limits and a full experiment is need to establish how a group of operators can cause fluctuations in the level of the OEE index. Finally, prior analysis of the data through the use of well-known techniques (such as CMLHL) would contribute to the evolution of MES design and engineering.

\section{Acknowledgements}

This research has been partially funded by the Spanish Ministry of Science and Innovation, under project TIN2008-06681-C06-04 and TIN201021272-C02-01, the Spanish Ministry of Science and Innovation through project PID 560300-2009-11, and project BU006A08 and CCTT/10/BU/0002 of the Junta de Castilla y León. The authors would also like to thank the manufacturer of vehicle interior components, Grupo Antolin Ingeniería, S.A., as part of the MAGNO 2008 - 1028.- CENIT Project funded by the Spanish Ministry of Science and Innovation.

\section{References}

[1] A. Abraham, E. Corchado and J. M. Corchado, Hybrid learning machines. Neurocomputing 72:13-15 (2009), 2729-2730.

[2] R. Acharya, E.C.P. Chua, K.C. Chua, L.C. Min, and T. Tamura. Analysis and Automatic Identification of Sleep Stages using Higher Order Spectra. International Journal of Neural Systems 20:6 (2010), 509-521.

[3] H. Adeli and S.L. Hung. An Adaptive Conjugate Gradient Learning Algorithm for Effective Training of Multilayer Neural Networks. Applied Mathematics and Computation 62:1 (1994), 81-102.
[4] H. Adeli and S.L. Hung. Machine Learning - Neural Networks, Genetic Algorithms, and Fuzzy Systems. Wiley \& Sons, New York, USA, 1995.

[5] H. Adeli and X. Jiang. Neuro-Fuzzy Logic Model for Freeway Work Zone Capacity Estimation. Journal of Transportation Engineering 129:5 (2003), 484-493.

[6] H. Adeli and X. Jiang. Dynamic fuzzy wavelet neural network model for structural system identification. Journal of Structural Engineering, ASCE, 132:1 (2006), 102-111.

[7] H. Adeli and A. Karim. Neural Network Model for Optimization of Cold-Formed Steel Beams. Journal of Structural Engineering, ASCE 123:11 (1997), 1535-1543.

[8] H. Adeli and A. Karim. Scheduling/Cost Optimization and Neural Dynamics Model for Construction. Journal of Construction Management and Engineering, ASCE 123:4 (1997), 450-458.

[9] H. Adeli and S. Kumar. Distributed Genetic Algorithms for Structural Optimization. Journal of Aerospace Engineering 8:3 (1995), 156-163.

[10] H. Adeli and S. Kumar. Concurrent Structural Optimization on a Massively Parallel Supercomputer. Journal of Structural Engineering, ASCE 121:11 (1995), 1588-1597.

[11] H. Adeli and H.S. Park. Counter Propagation Neural Network in Structural Engineering. Journal of Structural Engineering, ASCE 121:8 (1995), 1205-1212.

[12] H. Adeli and H.S. Park. A Neural Dynamics Model for Structural Optimization - Theory. Computers and Structures 57:3 (1995), 383-390.

[13] H. Adeli and H.S. Park. Optimization of Space Structures by Neural Dynamics. Neural Networks 8:5 (1995), 769-781.

[14] H. Adeli and A. Samant. An Adaptive Conjugate Gradient Neural Network - Wavelet Model for Traffic Incident Detection. Computer-Aided Civil and Infrastructure Engineering 15:4 (2000), 251-260.

[15] M. Ahmadlou and H. Adeli, H. Enhanced Probabilistic Neural Network with Local Decision Circles: A Robust Classifier, Integrated Computer-Aided Engineering 17:3 (2010), 197-210.

[16] A. Al-Bazi and N. Dawood. Developing Crew Allocation System for Precast Industry Using Genetic Algorithms. Computer-Aided Civil and Infrastructure Engineering 25:8 (2010), 581-595.

[17] J. Alcalá-Fdez, L. Sánchez, S. García, M.J. del Jesus, S. Ventura, J.M. Garrell, J. Otero, C. Romero, J. Bacardit, V.M. Rivas, J.C. Fernández, F. Herrera, KEEL: A Software Tool to Assess Evolutionary Algorithms to Data Mining Problems, Soft Computing 13:3 (2009), 307-318.

[18] Apriso Corporation, Manufacturing Execution Systems Strategy Update: Trends and Tips for 2010 , http://www.bitpipe.com/detail/RES/1268690867 382.html.

[19] S. Arangio and F. Bontempi. Soft Computing based Multilevel Strategy for Bridge Integrity Monitoring. ComputerAided Civil and Infrastructure Engineering 25:5 (2010), 348362.

[20] F. J. Berlanga, A. J. Rivera Rivas, M. J. del Jesús and F. Herrera, GP-COACH: Genetic Programming-based learning of COmpact and ACcurate fuzzy rule-based classification systems for High-dimensional problems. Information Science. 180:8 (2010), 1183-1200.

[21] A. Bianchini and P. Bandini. Prediction of Pavement Performance through Neuro-Fuzzy Reasoning. Computer-Aided Civil and Infrastructure Engineering. 25:1 (2010), 39-54.

[22] L. Carro-Calvo, S. Salcedo-Sanz, R. Gil-Pita, A. PortillaFigueras and M. Rosa-Zurera. An Evolutive Multiclass Algorithm for Automatic Classification of High Range Resolu- 
tion Radar Targets. Integrated Computer-Aided Engineering 16:1 (2009), 51-60.

[23] L. Carro-Calvo, S. Salcedo-Sanz, G. Ortiz-García and A. Portilla-Figueras. An Incremental-Encoding Evolutionary Algorithm for Color Reduction in Images. Integrated Computer-Aided Engineering 17:3 (2010), 261-269.

[24] S. le Cessie and J.C. van Houwelingen, Ridge Estimators in Logistic Regression, Applied Statistics 41:1 (1992), 191-201.

[25] R. S. Chen, Y. S. Tsai and C. C. Chang, Design and implementation of an intelligent manufacturing execution system for semiconductor manufacturing industry, Proceedings of the 2006 IEEE International Symposium on Industrial Electronics (2006), 2948-2953.

[26] T.M. Cheng and R.Z. Yan. Integrating Messy Genetic Algorithms and Simulation to Optimize Resource Utilization. Computer-Aided Civil and Infrastructure Engineering 24:6 (2009), 401-415.

[27] B. K. Choi and B. H. Kim, MES (Manufacturing Execution System) architecture for FMS compatible to ERP (Enterprise Planning System), International Journal of Computer Integrated Manufacturing 15:3 (2002), 274-284.

[28] S. Y. Chou, C. C. Chou, Y. K. and Chen, A Fundamental Tool Path Planning Problem for Circles in Layered Manufacturing, Integrated Computer-Aided Engineering, 15:1 (2008), 37-52.

[29] M. Coelho, A. Ferreira de Carvalho, R. J. Gabrielli B. Campello and E. R. Hruschka, Genetic Clustering for Data Mining. Soft Computing for Knowledge Discovery and Data Mining (2008), 113-132.

[30] E. Corchado and C. Fyfe, Connectionist techniques for the identification and suppression of interfering underlying factors, International Journal of Pattern Recognition and Artificial Intelligence 17:8 (2003), 1447-1466.

[31] E. Corchado and A. Hererro, Neural Visualization of Network Traffic Data for Intrusion Detection. Applied Soft Computing 11:2 (2011), 2042-2056.

[32] E. Corchado and P. Novais, Soft Computing Models in Industrial and Environmental Applications, Proceedings of the 5th International Workshop (SOCO 2010). Springer Series: Advances in Intelligent and Soft Computing, Vol. 73, 2010.

[33] T.M. Cover and P.E. Hart, Nearest Neighbor Pattern Classification, IEEE Transactions on Information Theory 13 (1967), 21-27.

[34] C. Cruz and D. Pelta, Soft computing and cooperative strategies for optimization. Applied. Soft Computing 9:1 (2009), 30-38.

[35] S. Das, A. Abraham and A. Konar, Automatic kernel clustering with a Multi-Elitist Particle Swarm Optimization Algorithm. Pattern Recognition Letters 29:5 (2008), 688-699.

[36] H. Duan, C.F. Xu and Z.H. Xing. A Hybrid Artificial Bee Colony Optimization and Quantum Evolutionary Algorithm for Continuous Optimization Problems. International Journal of Neural Systems 20:1 (2010), 39-50.

[37] H.M. Elragal. Improving Neural Networks Prediction accuracy Using Particle Swarm Optimization Combiner. International Journal of Neural Systems 19:5 (2009), 387-393.

[38] Salvador García and Francisco Herrera, An Extension on "Statistical Comparisons of Classifiers over Multiple Data Sets" for all Pair wise Comparisons, Journal of Machine Learning Research 9 (2008), 2677-2694.

[39] S. Ghosh-Dastidar and H. Adeli. Wavelet-Clustering-Neural Network Model for Freeway Incident Detection. ComputerAided Civil and Infrastructure Engineering 18:5 (2003), 325338

[40] S. Ghosh-Dastidar and H. Adeli. Neural Network-Wavelet Micro-Simulation Model for Delay and Queue Length Esti- mation at Freeway Work Zones. Journal of Transportation Engineering, ASCE 132:4 (2006), 331-341.

[41] S. Ghosh-Dastidar and H. Adeli. Improved Spiking Neural Networks for EEG Classification and Epilepsy and Seizure Detection. Integrated Computer-Aided Engineering 14:3 (2007), 187-212.

[42] S. Ghosh-Dastidar, H. Adeli, and N. Dadmehr. Principal component analysis-enhanced cosine radial basis function neural network for robust epilepsy and seizure detection. IEEE Transactions on Biomedical Engineering, 55:2 (2008), 512-518.

[43] W. Graf, S. Freitag, M. Kaliske, and J.U. Sickert. Recurrent neural networks for uncertain time-dependent structural behavior. Computer-Aided Civil and Infrastructure Engineering 25:5 (2010), 322-333.

[44] A.M.A. Haidar, A. Mohamed, M. Al-Dabbagh, A. Aini Hussain and M. Masoum. An Intelligent Load Shedding Scheme Using Neural networks \& Neuro-Fuzzy. International Journal of Neural Systems 19:6 (2009), 473-479.

[45] M. Hall, E. Frank, G. Holmes, B. Pfahringer, P. Reutemann and I. H. Witten. The WEKA Data Mining Software: An Update; SIGKDD Explorations, Volume 11, Issue 1 (2009).

[46] F. Herrera and J. L. Verdegay, Genetic Algorithms and Soft Computing (Studies in Fuzziness and Soft Computing), Physica-Verlag, 1997.

[47] A. Herrero, E. Corchado, L. Sáiz and A. Abraham, DIPKIP: A Connectionist Knowledge Management System to Identify Knowledge Deficits in Practical Cases. Computational Intelligence 26:1 (2010), 26-56.

[48] S.L Hung and H. Adeli. Parallel Backpropagation Learning Algorithms on Cray Y-MP8/864 Supercomputer. Neurocomputing 5:6 (1993), 287-302.

[49] S.L. Hung and H. Adeli. A Parallel Genetic/Neural Network Learning Algorithm for MIMD Shared Memory Machines. IEEE Transactions on Neural Networks 5:6 (1994), 900-909.

[50] J.A. Iglesias, P. Angelov, A. Ledezma and A. Sanchis. Human Activity Recognition Based on Evolving Fuzzy Systems. International Journal of Neural Systems 20:5 (2010), 355364.

[51] Laboratory of Artificial Intelligence, University of Ljubljana, Orange Machine Learning Library, http://orange.biolab.si/

[52] H. Ishibuchi, T. Yamamoto and T. Nakashima, Hybridization of Fuzzy GBML Approaches for Pattern Classification Problems, IEEE Transactions on Systems, Man and Cybernetics Part B: Cybernetics 35:2 (2005), 359-365.

[53] M. J. del Jesus, F. Hoffmann, L. Junco and L. Sánchez, Induction of Fuzzy-Rule-Based Classifiers With Evolutionary Boosting Algorithms, IEEE Transactions on Fuzzy Systems 12:3 (2004), 296-308.

[54] X. Jiang and H. Adeli. Fuzzy Clustering Approach for Accurate Embedding Dimension Identification in Chaotic Time Series. Integrated Computer-Aided Engineering 10:3 (2003), 287-302.

[55] X. Jiang and H. Adeli. Pseudospectra, MUSIC, and Dynamic Wavelet Neural Network for Damage Detection of Highrise Buildings. International Journal for Numerical Methods in Engineering 71:5 (2007), 606-629.

[56] X. Jiang and H. Adeli. Dynamic Fuzzy Wavelet Neuroemulator for Nonlinear Control of Irregular Highrise Building Structures. International Journal for Numerical Methods in Engineering 74:7 (2008), 1045-1066.

[57] X. Jiang and H. Adeli. Neuro-Genetic Algorithm for Nonlinear Active Control of Highrise Buildings. International Journal for Numerical Methods in Engineering 75:8 (2008), 770786 
[58] X.L. Jin and H. Doloi. Modelling Risk Allocation DecisionMaking in PPP Projects Using Fuzzy Logic. ComputerAided Civil and Infrastructure Engineering 24:7 (2009), 509524

[59] S.P. Johnston, G. Prasad, L. Maguire, and T.M. McGinnity. An FPGA Hardware/software co-design methodology - towards evolvable spiking networks for robotics application. International Journal of Neural Systems, 20:6 (2010), 447461

[60] M.W. Kang, P. Schonfeld and N. Yang. Prescreening and Repairing in a Genetic Algorithm for Highway Alignment Optimization. Computer-Aided Civil and Infrastructure Engineering 24:2 (2009), 109-119.

[61] A. Karim and H. Adeli. CONSCOM: An OO Construction Scheduling and Change Management System. Journal of Construction Engineering and Management 125:5 (1999), 368-376.

[62] A. Karim and H. Adeli. Object-Oriented Information Model for Construction Project Management. Journal of Construction Engineering and Management, ASCE 125:5 (1999), 361-367.

[63] A. Karim and H. Adeli. Comparison of the Fuzzy - Wavelet RBFNN Freeway Incident Detection Model with the California Algorithm. Journal of Transportation Engineering. 128:1 (2002), 21-30.

[64] A. Karim and H. Adeli. Radial Basis Function Neural Network for Work Zone Capacity and Queue Estimation. Journal of Transportation Engineering 129:5 (2003), 494-503.

[65] A. Karim and H. Adeli. CBR Model for Freeway Work Zone Traffic Management. Journal of Transportation Engineering. Journal of Transportation Engineering, ASCE 129:2 (2003), 134-145.

[66] KDnuggets, Data Mining Community's top resources: Software Suites for Data Mining, Analytics, and Knowledge Discovery, http://www.kdnuggets.com/software/suites.html

[67] H. Kim and H. Adeli. Discrete Cost Optimization of Composite Floors using a Floating Point Genetic Algorithm. Engineering Optimization 33:4 (2001), 485-501.

[68] Y. Kim, R. Langari and S. Hurlebus. Model-based Multiinput, Multi-output Supervisory Semiactive Nonlinear Fuzzy Controller. Computer-Aided Civil and Infrastructure Engineering 25:5 (2010), 387-393.

[69] P. Koprinkova-Hriatova. Backpropagation through Time Training of a Neuro-fuzzy Controller. International Journal of Neural Systems 20:5 (2010), 421-428.

[70] Y. Lee and C.H. Wei. A Computerized Feature Selection Using Genetic Algorithms to Forecast Freeway Accident Duration Times. Computer-Aided Civil and Infrastructure Engineering 25:2 (2010), 132-148.

[71] X. Li, M.J. Er, B.S. Lim, J.H. Zhou, O.P. Gan and L. Rutkowski. Fuzzy Regression Modelling for Tool Performance Prediction and Degradation Detection. International Journal of Neural Systems 20:5 (2010), 405-419.

[72] A. C. Lorena, A. Ferreira de Carvalho, Evolutionary Design of Code-matrices for Multiclass Problems. Soft Computing for Knowledge Discovery and Data Mining (2008), 153-184.

[73] M. Martínez-Ballesteros, A. Troncoso, F. Martínez-Álvarez and J.C. Riquelm. Mining Quantitative Association Rules Based on Evolutionary Computation and its Application to Atmospheric Pollution. Integrated Computer-Aided Engineering 17:3 (2010), 227-242.

[74] M. McClellan, Introduction to Manufacturing Execution Systems, Proceedings of MES Conference and Exposition, USA 2001.

[75] G. J. McLachlan, Discriminant Analysis and Statistical Pattern Recognition, John Wiley and Sons, 2004.
[76] E. Nichols, L.J. McDaid and N.H. Siddique. Case Study on Self-organizing Spiking Neural Networks for Robot Navigation. International Journal of Neural Systems 20:6 (2010), 501-508.

[77] A. Panakkat and H. Adeli. Recurrent Neural Network for Approximate Earthquake Time and Location Prediction Using Multiple Seismicity Indicators. Computer-Aided Civil and Infrastructure Engineering 24:4 (2009), 280-292.

[78] H.S. Park and H. Adeli. A Neural Dynamics Model for Structural Optimization - Application to Plastic Design of Structures. Computers and Structures 57:3 (1995), 391-399.

[79] H.S. Park and H. Adeli. Distributed Neural Dynamics Algorithms for Optimization of Large Steel Structures. Journal of Structural Engineering, ASCE 123:7 (1997), 880-888.

[80] P.B. Patel and T. Marwala. Caller behaviour classification using Computational Intelligence Methods. International Journal of Neural Systems 20:1 (2010), 87-93.

[81] P. Patrinos, A. Alexandridis, K. Ninos and H. Sarimveis. Variable Selection in Nonlinear Modeling based on RBF Networks and Evolutionary Computation. International Journal of Neural Systems 20:5 (2010), 365-379.

[82] M. Prymek and A. Horak. Multi-agent Approach to Power Distribution Network Modelling. Integrated Computer-Aided Engineering 17:4 (2010), 291-303.

[83] G. Puscasu and B. Codres. Nonlinear System Identification Based on Internal Recurrent Neural Networks. International Journal of Neural Systems 19:2 (2009), 115-125.

[84] J. R. Quinlan, C4.5: Programs for Machine Learning, Morgan Kauffman, 1993.

[85] J.R. Quinlan, MDL and Categorical Theories (Continued). Machine Learning: Proceedings of the Twelfth International Conference. Lake Tahoe California (United States of America, (1995), 464-470.

[86] F. Rasheed, M. Alshalalfa and R. Alhajj. Adapting Machine Learning Technique for Periodicity Detection in Biological Sequences. International Journal of Neural Systems 19:1 (2009), 11-24.

[87] U. Reuter and B. Moeller. Artificial Neural Networks for Forecasting of Fuzzy Time Series. Computer-Aided Civil and Infrastructure Engineering 25:5 (2010), 363-374.

[88] A. Rodriguez and J. Reggia, A Distributed Learning Algorithm for Particle Systems, Integrated Computer-Aided Engineering, 16:1 (2009), 1-20.

[89] C. Rodríguez Valdez, R.M. Tallam, R.J. Kerkman, A Novel Frequency-Dependent-Model for Electric Conductors. Integrated Computer-Aided Engineering 16:1 (2009), 105-117.

[90] S. Rokni and A. R. Fayek, A Multi-Criteria Optimization Framework for Industrial Shop Scheduling Using Fuzzy Set Theory, Integrated Computer-Aided Engineering, 17:3 (2010), pp. 175-196.

[91] J.L. Rossello, V. Canals, A. Morro and J. Verd. Chaos-based Mixed Signal Implementation of Spiking Neurons. International Journal of Neural Systems 19:6 (2009), 465-471.

[92] A. Samant and H. Adeli. Enhancing Neural Network Incident Detection Algorithms using Wavelets. Computer-Aided Civil and Infrastructure Engineering 16:4 (2001), 239-245.

[93] L. Sánchez, I. Couso and J.A. Corrales, Combining GP Operators With SA Search To Evolve Fuzzy Rule Based Classifiers, Information Sciences 136:1-4 (2001), 175-192.

[94] M.J. Santofimia, X. del Toro, P. Roncero, F. Moya, M.A. Martinez and J.C. Lopez. A Qualitative Agent-based Approach to Power Quality Monitoring and Diagnosis. Integrated Computer-Aided Engineering 17:4 (2010), 305-319.

[95] K. Sarma and H. Adeli. Fuzzy Genetic Algorithm for Optimization of Steel Structures. Journal of Structural Engineering, ASCE 126:5 (2000), 596-604. 
[96] K. Sarma and H. Adeli. Fuzzy Discrete Multicriteria Cost Optimization of Steel Structures. Journal of Structural Engineering, ASCE 126:11 (2000), 1339-1347.

[97] K.C. Sarma and H. Adeli. Bi-Level Parallel Genetic Algorithms for Optimization of Large Steel Structures. ComputerAided Civil and Infrastructure Engineering 16:5 (2001), 295304

[98] K.C. Sarma and H. Adeli. Life-Cycle Cost Optimization of Steel Structures. International Journal for Numerical Methods in Engineering 55:12 (2002), 1451-1462.

[99] S. Schliebs, N. Kasabv, and M. Defoin-Platel. On the Probabilistic Optimization of Spiking Neural Networks. International Journal of Neural Systems 20:6 (2010), 481-500.

[100]R. Scherer. Designing Boosting Ensemble of Relational Fuzzy Systems. International Journal of Neural Systems 20:5 (2010), 381-388.

[101] J. Sedano J, E. Corchado, L. Curiel, J.R. Villar, E. de la Cal. Detection of heat flux failures in building using a soft computing diagnostic system. Neural Network World 20:7 (2010), 883-898.

[102] J. Sedano, J. R. Villar, E. S. Corchado, L. Curiel and P. M. Bravo, Modelling a Pneumatic Drill Process by a two-steps AI Model, International Journal of Computer Mathematics 86:10-11 (2009), 1769-1777.

[103] J. Sedano, L. Curiel, E. Corchado, E. de la Cal and J. R. Villar, A soft computing method for detecting lifetime building thermal insulation failures, Integrated Computer-Aided Engineering 10:2 (2010), 103-115.

[104] A.B. Senouci and H. Adeli. Resource Scheduling using Neural Dynamics Model of Adeli and Park. Journal of Construction Engineering and Management, ASCE 127:1 (2001), 2834.

[105] G.F. Sirca Jr. and H. Adeli. Case-Based Reasoning for Converting Working Stress Design-Based Bridge Ratings to Load Factor Design-Based Ratings. Journal of Bridge Engineering 10: 4 (2005), 450-459.

[106] S. Soltic and N. Kasabov. Knowledge extraction from evolving spiking neural networks with rank order population coding. International Journal of Neural Systems 20:6 (2005), 437-445.

[107] T.J. Strain, L.J. McDaid, L.P. Maguire, and T.M. McGinnity. An STDP Training Algorithm for a Spiking Neural Network with Dynamic Threshold Neurons. International Journal of Neural Systems 20:6 (2010), 463-480.

[108]L. Tan and S. Shenghan Xu. A Model-Checking-Based Approach to Risk Analysis in Supply Chain Consolidations. Integrated Computer-Aided Engineering 16:3 (2009), 243-257.

[109] A.R. Tashakori and H. Adeli. Optimum Design of ColdFormed Steel Space Structures Using Neural Dynamic Mod- el. Journal of Constructional Steel Research 58:12 (2001), 1545-1566.

[110] D.C. Theodoridis, Y.S. Boutalis and M.A. Christodoulou. Indirect Adaptive Control of Unknown Multi Variable Nonlinear Systems with Parametric and Dynamic Uncertainties Using a New Neuro-Fuzzy System Description. International Journal of Neural Systems 20:2 (2010), 129-148.

[111] V. Torra and Y. Narukawa, Soft Computing in decision modelling. Soft Computing 14:5 (2010), 419-420.

[112]B. S. de Ugarte, A. Artiba and R. Pellerin, Manufacturing execution system - a literature review, Production planning and control 20:6 (2009), 525-539.

[113] L. Van Dyk, Manufacturing execution systems, Master Thesis in Engineering dissertation, University of Pretoria, Pretoria, http://upetd.up.ac.za/thesis/available/etd-11092006125332/, (1999).

[114] J.R. Villar, E. de la Cal and J. Sedano. A Fuzzy Logic Based Efficient Energy Saving Approach for Domestic Heating Systems, Integrated Computer-Aided Engineering 16: 2 (2009), 151-163.

[115]B.J. Vitins and K.W. Axhausen. Optimization of Large Transport Networks Using the Ant Colony Heuristic. Computer-Aided Civil and Infrastructure Engineering 24:1 (2009), $1-14$.

[116] A. Waheed and H. Adeli. Case-Based Reasoning in Steel Bridge Engineering. Knowledge-Based Systems 18:1 (2005), 37-46.

[117] N. Wang, M. Joo, X.Y. Meng and X. Li. An Online Selforganizing Scheme for Parsimonious and Accurate Fuzzy Neural Networks. International Journal of Neural Systems 20:5 (2010), 389-403.

[118]Z. Ye and Y. Zhang. Speed Estimation from Single Loop Data Using an Unscented Particle Filter. Computer-Aided Civil and Infrastructure Engineering 25:7 (2010), 494-503.

[119] G. Yu and H. Adeli. Object-Oriented Finite Element Analysis Using EER Model. Journal of Structural Engineering, ASCE 119:9 (1993), 2763-2781.

[120] W. Yu and X. Li. Automated Nonlinear System Modeling with Multiple Fuzzy Neural Networks and Kernel Smoothing. International Journal of Neural Systems 20:5 (2010), 429435

[121] L. A. Zadeh, Towards extended fuzzy logic - A first step. Fuzzy Sets and Systems 160:21 (2009), 3175-3181.

[122] J.A. Zeferino, A.P. Antunes and M.C. Cunha. An Efficient Simulated Annealing Algorithm for Regional Wastewater Systems Planning. Computer-Aided Civil and Infrastructure Engineering 24:5 (2009), 359-370. 